TRANSACTIONS OF THE

AMERICAN MATHEMATICAL SOCIETY

Volume 362, Number 3, March 2010, Pages 1211-1230

S 0002-9947(09)04774-6

Article electronically published on October 9, 2009

\title{
MIXED BOUNDARY VALUE PROBLEMS FOR THE STOKES SYSTEM
}

\author{
R. BROWN, I. MITREA, M. MITREA, AND M. WRIGHT
}

Dedicated to the memory of Misha Cotlar

\begin{abstract}
We prove the well-posedness of the mixed problem for the Stokes system in a class of Lipschitz domains in $\mathbb{R}^{n}, n \geq 3$. The strategy is to reduce the original problem to a boundary integral equation, and we establish certain new Rellich-type estimates which imply that the intervening boundary integral operator is semi-Fredholm. We then prove that its index is zero by performing a homotopic deformation of it onto an operator related to the Lamé system, which has recently been shown to be invertible.
\end{abstract}

\section{INTRODUCTION}

Given an open set $\Omega \subset \mathbb{R}^{n}$ with outward unit normal $\vec{\nu}$, along with a partition $D, N$ of $\partial \Omega$, consider the mixed boundary value problem for the Stokes system

$$
\left\{\begin{array}{l}
\Delta \vec{u}-\nabla \pi=0 \text { and } \operatorname{div} \vec{u}=0 \text { in } \Omega, \\
\left.\vec{u}\right|_{D}=\vec{f}_{D} \in L_{1}^{2}(D), \\
{\left.\left[\left[\nabla \vec{u}+(\nabla \vec{u})^{\top}\right] \vec{\nu}-\pi \vec{\nu}\right]\right|_{N}=\vec{f}_{N} \in L^{2}(N),} \\
M(\nabla \vec{u}), M(\vec{u}), M(\pi) \in L^{2}(\partial \Omega),
\end{array}\right.
$$

where $\vec{f}_{D}, \vec{f}_{N}$ are two given functions. Hereafter, $M$ denotes the nontangential maximal operator, and boundary traces are taken in the sense of pointwise nontangential limits. Finally, $L^{2}(N)$ is the Lebesgue space of square-integrable functions (with respect to the surface measure) on $N$, whereas $L_{1}^{2}(D)$ stands for the Sobolev space of order one in $D$. See Section 2 for more details.

The vector field $\vec{u}=\left(u_{1}, \ldots, u_{n}\right)$ and scalar-valued function $\pi$ represent, respectively, the velocity and the pressure of the fluid. In (1.1), $\nabla \vec{u}:=\left(\partial u_{j} / \partial x_{k}\right)_{1 \leq j, k \leq n}$ is the Jacobian matrix of $\vec{u}$, and the superscript $T$ indicates transposition. Such problems arise when modeling the behavior of an incompressible medium in a rigid open container (such is the case with the water flowing in a river when $D$ is the surface of the river bed and $N$ is the free surface of the water). See, e.g., [11, 13 and the references therein.

Received by the editors June 25, 2007.

2000 Mathematics Subject Classification. Primary 35J25, 42B20; Secondary 35J05, 45B05, $31 \mathrm{~B} 10$.

Key words and phrases. Stokes system, Lipschitz domains, mixed boundary value problems, layer potentials, well-posedness.

The research of the authors was supported in part by the NSF. 
Let us now describe the class of domains in which we shall study (1.1). To begin with we shall assume that $\Omega \subseteq \mathbb{R}^{n}, n \geq 3$, is a bounded Lipschitz domain with connected boundary. Being a Lipschitz domain in $\mathbb{R}^{n}$ amounts to having a boundary which locally coincides (up to a rigid motion of the space) with the graph of a real-valued Lipschitz function defined in $\mathbb{R}^{n-1}$; cf. Definition 2.1. In addition, the boundary $\partial \Omega$ is partitioned into two pieces $N, D$ which are assumed to meet at an angle $<\pi$, roughly speaking. Such domains will be referred to as being creased Lipschitz domains; cf. Definition 2.2. They were first considered in [3, where it was shown that the mixed problem with $L^{2}$-data for Laplace's equation is well-posed in such domains. In particular, this offered a partial solution to a question posed by C. Kenig on p. 120 of 12 .

The case of the the mixed problem for the Lamé differential operator,

$$
\left\{\begin{array}{l}
\mu \Delta \vec{u}+(\mu+\lambda) \nabla(\operatorname{div} \vec{u})=0 \text { in } \Omega, \\
\left.\vec{u}\right|_{D}=\vec{f}_{D} \in L_{1}^{2}(D), \\
{\left.\left[\mu\left[\nabla \vec{u}+(\nabla \vec{u})^{\top}\right] \vec{\nu}+\lambda(\operatorname{div} \vec{u}) \vec{\nu}\right]\right|_{N}=\vec{f}_{N} \in L^{2}(N),} \\
M(\nabla \vec{u}), M(\vec{u}) \in L^{2}(\partial \Omega),
\end{array}\right.
$$

with

$$
\mu>0 \text { and } \lambda \geq-\frac{2 \mu}{n},
$$

has been recently treated in [4] once again, when $D, N$ form a creased partition of the boundary of the Lipschitz domain $\Omega \subset \mathbb{R}^{n}, n \geq 3$.

The well-posedness result pertaining to (1.2) is relevant to the present work since our strategy for dealing with (1.1) can be summarized as follows. First, we show that the mixed problem for the Stokes system can be reduced to the issue of inverting a certain boundary integral operator $T$. Second, we develop a new set of Rellich-type estimates which, in turn, allow us to show that $T$ is an injective, semi-Fredholm operator. Thus, matters are reduced to proving that $T$ has index zero. Parallel considerations work in the case of (1.2) when, once again, the wellposedness of the problem in question is equivalent to inverting a certain boundary singular integral operator, called $T_{\mu, \lambda}$. The main result in [4] implies that $T_{\mu, \lambda}$ is invertible whenever (1.3) holds. The somewhat unexpected link between (1.2) and (1.1), seen at the level of these boundary singular integral operators, is that

$$
\lim _{\lambda \rightarrow \infty} T_{1, \lambda}=T
$$

in the strong, operator norm. Consequently, the a priori knowledge that $T$ is semiFredholm allows us to deduce that index $(T)=\operatorname{index}\left(T_{1, \lambda}\right)=0$, and the desired conclusion about (1.1) follows.

This is interesting inasmuch as the limiting case of the Lamé system $\mu \Delta+$ $(\mu+\lambda) \nabla \operatorname{div}$ as $\lambda \rightarrow \infty$ is not the Stokes system. Let us also point out that, in hindsight, it is natural that in the chronology of mixed problems in Lipschitz domains, the Stokes system has been treated after the Lamé system and Laplacian, since the respective solutions make use of earlier work in precisely this order. As expected, the Stokes system is also somewhat special due to the relatively unorthodox role played by the pressure function.

Our main result regarding (1.1) reads as follows. 
Theorem 1.1. Let $\Omega$ be a bounded, creased, Lipschitz domain in $\mathbb{R}^{n}, n \geq 3$, with connected boundary. Then there exists $\varepsilon>0$ such that the mixed boundary value problem (1.1) has a unique solution $\vec{u}$ for any given $\vec{f}_{D} \in L_{1}^{2}(D), \vec{f}_{N} \in L^{2}(N)$. In addition, this solution satisfies

$$
\begin{aligned}
& \|M(\nabla \vec{u})\|_{L^{2}(\partial \Omega)}+\|M(\vec{u})\|_{L^{2}(\partial \Omega)}+\|M(\pi)\|_{L^{2}(\partial \Omega)} \\
& \leq C\left\|\vec{f}_{D}\right\|_{L_{1}^{2}(D)}+C\left\|\vec{f}_{N}\right\|_{L^{2}(N)},
\end{aligned}
$$

(where $C$ depends only on $\Omega, D$ and $N$ ) and can be represented in terms of layer potentials associated with the Stokes system.

This can be regarded as an extension of the main results pertaining to the Stokes system in [7, 9], where the limiting cases when either $D$ or $N$ is empty have been treated.

In fact, our methods can handle various other versions and generalizations of this result. For example, Theorem 5.4 treats the case of a graph Lipschitz domain (while retaining the assumption that $D, N$ form a creased partition of $\partial \Omega$ ). Also, in Theorem 6.3, we prove a well-posedness result for the mixed problem for the Stokes system in bounded, creased Lipschitz domains in $\mathbb{R}^{n}$, in the case when the boundary condition involving the traction is replaced by

$$
\left.\left[\left[r(\nabla \vec{u})+(\nabla \vec{u})^{\top}\right] \vec{\nu}-\pi \vec{\nu}\right]\right|_{N}=\vec{f}_{N} \in L^{p}(N)
$$

where $r \in(-1,1]$ is a parameter. Of course, $r=1$ corresponds to (1.1), which is the most physically relevant case. However, not only does this formalism allow for the simultaneous consideration (and economical treatment) of a wide spectrum of conormal derivatives for the Stokes system (e.g., the case $r=0$ corresponds to the conormal derivative used in 9]), but the consideration of (1.6) turns out to be (as is apparent from the considerations in Section 4) very useful even if one is only interested in (1.1) alone.

Our approach, based on boundary layer potentials and Calderón-Zygmund theory, along with well-understood perturbation techniques, allows us in fact to show that the $L^{p}$-version of (1.1) remains well-posed whenever $|p-2|<\varepsilon$, for some small $\varepsilon=\varepsilon(\Omega, D, N)>0$. While determining the optimal $L^{p}$-solvability range for the mixed problem (1.1) remains open at the moment, we wish to point out that recent progress in the case when $N=\emptyset$ (corresponding to the so-called regularity problem) and $D=\emptyset$ (corresponding to the Neumann problem) for the Stokes system in an arbitrary Lipschitz domain $\Omega \subset \mathbb{R}^{n}, n \geq 2$, has been made in [21.

For more on the topic of mixed boundary problems in Lipschitz domains the interested reader is referred to [3, [14, [16, 18, 20, and the references cited therein. Let us also mention [15, where value problems for the Navier-Stokes system in polyhedral domains with a variety of mixed boundary conditions are studied and where references to earlier work on this topic can be found.

The layout of the current paper is as follows. In Section 2 we collect a number of rudiments regarding the geometry of creased Lipschitz domains, function spaces, the algebraic structure of the Stokes system, and set up the associated layer potentials. In Section 3, we take the first step in the treatment of the mixed problem for creased graph Lipschitz domains by reducing this problem to a boundary integral equation. Section 4 is largely devoted to developing a new set of Rellich-type estimates which are key to our subsequent analysis (see also the comments made at the beginning of Section 4). This is the main technical innovation of this paper. Armed with 
the aforementioned estimates, we finish in Section 5 the proof of the well-posedness of the mixed problem in the graph case. The main novelty here is the use of a homotopy linking the layer potentials associated, on the one hand, with Stokes system, with those associated, on the other hand, with the Lamé system. For this segment in our analysis, the results in [4 play a basic role. Finally, in Section 6, we indicate how this technology can be adapted to the case of bounded creased Lipschitz domains.

\section{Preliminaries}

We start by reviewing certain geometrical concepts.

Definition 2.1. Call an open set $\Omega \subset \mathbb{R}^{n}$ a Lipschitz domain, provided there exists a finite $M>0$ for which the following is true. For every $x \in \partial \Omega$ there exists a coordinate system in $\mathbb{R}^{n}$ (obtained from the standard one via a rigid motion) say, $\left(x^{\prime}, x_{n}\right)=\left(x_{1}, x^{\prime \prime}, x_{n}\right) \in \mathbb{R} \times \mathbb{R}^{n-2} \times \mathbb{R}$, a cylinder $C_{r}(x):=\left\{\left(y^{\prime}, y_{n}\right):\left|y^{\prime}-x^{\prime}\right|<\right.$ $\left.r,\left|y_{n}-x_{n}\right|<2 M r\right\}$ for some $r>0$, and a Lipschitz function $\phi: \mathbb{R}^{n-1} \rightarrow \mathbb{R}$ with $\|\nabla \phi\|_{L^{\infty}\left(\mathbb{R}^{n-1}\right)} \leq M$, such that

$$
\begin{aligned}
& C_{r}(x) \cap \Omega=\left\{\left(y^{\prime}, y_{n}\right): y_{n}>\phi\left(y^{\prime}\right)\right\} \cap C_{r}(x), \\
& C_{r}(x) \cap \partial \Omega=\left\{\left(y^{\prime}, y_{n}\right): y_{n}=\phi\left(y^{\prime}\right)\right\} \cap C_{r}(x) .
\end{aligned}
$$

If $\partial \Omega$ can be described using just one coordinate cylinder, we shall refer to $\Omega$ as a graph Lipschitz domain. As is well known, any Lipschitz domain $\Omega \subset \mathbb{R}^{n}$ has a well-defined surface measure $\sigma$ and outward unit normal $\vec{\nu}(x)$ at $\sigma$-a.e. $x \in \partial \Omega$.

Definition 2.2. Let $\Omega$ be a bounded Lipschitz domain in $\mathbb{R}^{n}$ with connected boundary $\partial \Omega$. Assume that $\partial \Omega=\bar{D} \cup \bar{N}$, where $D, N \subseteq \partial \Omega$ are nonempty open sets with $D \cap N=\emptyset$. In addition, it is assumed that there exists a finite constant $m>0$ with the property that for each $x \in \bar{D} \cap \bar{N}$ there exists a Lipschitz function $\psi: \mathbb{R}^{n-2} \rightarrow \mathbb{R}$ and some $r>0$ satisfying (with $C_{r}(x)$ and $\phi$ as in (2.1)):

(i) $N \cap C_{r}(x)=C_{r}(x) \cap \partial \Omega \cap\left\{\left(x_{1}, x^{\prime \prime}, x_{n}\right): x_{1} \geq \psi\left(x^{\prime \prime}\right)\right\}$;

(ii) $D \cap C_{r}(x)=C_{r}(x) \cap \partial \Omega \cap\left\{\left(x_{1}, x^{\prime \prime}, x_{n}\right): x_{1}<\psi\left(x^{\prime \prime}\right)\right\}$;

(iii) $\frac{\partial \phi}{\partial x_{1}}>m$ a.e. for $x_{1}>\psi\left(x^{\prime \prime}\right)$ and $\frac{\partial \phi}{\partial x_{1}}<-m$ a.e. for $x_{1}<\psi\left(x^{\prime \prime}\right)$.

The reason for the terminology employed here is that it is reasonable to think of the interface $\bar{D} \cap \bar{N}$ as a 'crease' for $\Omega$. Heuristically, this is a quantified way of expressing the fact that $D$ and $N$ meet at an angle $<\pi$. As simple counterexamples indicate (cf., e.g., the discussion on p. 1231 in [3] ), such a property is quite natural, in fact necessary, in the context of mixed boundary problems.

A distinguished feature of a creased Lipschitz domain (bounded or graph) is the existence of a vector field $\vec{h} \in C_{0}^{\infty}\left(\mathbb{R}^{n}\right)$ which satisfies

$$
\langle\vec{h}, \vec{\nu}\rangle>\delta \quad \text { a.e. on } N \quad \text { and } \quad\langle\vec{h}, \vec{\nu}\rangle<-\delta \quad \text { a.e. on } D \text {. }
$$

See [3], 4], for discussions.

Assume next that a Lipschitz domain $\Omega \subset \mathbb{R}^{n}$ with outward unit normal $\vec{\nu}=$ $\left(\nu_{j}\right)_{1 \leq j \leq n}$ and surface measure $\sigma$ has been fixed. Given a scalar-valued function $u$, we define the tangential gradient of $u$ on $\partial \Omega$ by setting

$$
\nabla_{\tan } u:=\left(\nu_{k} \partial_{\tau_{k j}} u\right)_{1 \leq j \leq n},
$$


where

$$
\partial_{\tau_{j k}} u:=\left.\nu_{k}\left(\partial_{j} u\right)\right|_{\partial \Omega}-\left.\nu_{j}\left(\partial_{k} u\right)\right|_{\partial \Omega}, \quad j, k=1, \ldots, n .
$$

In particular, $\partial_{\tau_{j k}} u=\nu_{j}\left(\nabla_{\tan } u\right)_{k}-\nu_{k}\left(\nabla_{\tan } u\right)_{j}$ for $j, k=1, \ldots, n$, so that $\left|\nabla_{\tan } u\right| \approx$ $\sum_{j, k=1}^{n}\left|\partial_{\tau_{j k}} u\right|$. Finally, agree that $\nabla_{t a n}$ acts on vector fields component-wise.

Fix $p \in(1, \infty)$ arbitrary and $\Omega \subset \mathbb{R}^{n}$ Lipschitz. We then define $L^{p}$-based Sobolev-like spaces on an open subset $E$ of $\partial \Omega$ by setting

$L^{p}(E):=\left\{f: E \rightarrow \mathbb{R}: f\right.$ is measurable and $\left.\|f\|_{L^{p}(E)}:=\left(\int_{E}|f|^{p} d \sigma\right)^{1 / p}<+\infty\right\}$,

$L_{1}^{p}(E):=\left\{f \in L^{p}(E): \nabla_{t a n} f \in L^{p}(E)\right\}$,

$\dot{L}_{1}^{p}(E):=\left\{f \in L_{l o c}^{1}(E): \nabla_{\text {tan }} f \in L^{p}(E)\right\}$,

where $L_{l o c}^{1}(E)$ stands for the Lebesgue space of locally integrable functions on $E$, and equip the last two spaces with the norms

$$
\|f\|_{L_{1}^{p}(E)}:=\|f\|_{L^{p}(E)}+\left\|\nabla_{\text {tan }} f\right\|_{L^{p}(E)} \quad \text { and } \quad\|f\|_{\dot{L}_{1}^{p}(E)}:=\left\|\nabla_{\tan } f\right\|_{L^{p}(E)} .
$$

We shall, in fact, employ the same notation for the versions of these spaces corresponding to vector-valued functions. We will, nonetheless, use an arrow to indicate that a particular function is vector-valued.

Next, given $w: \Omega \rightarrow \mathbb{R}$, the nontangential maximal function of $w$ evaluated at $x \in \partial \Omega$ is defined as

$$
M(w)(x):=\sup \{|w(y)|: y \in \Gamma(x)\},
$$

where $\Gamma(x)$ stands for the nontangential approach region with vertex at $x \in \partial \Omega$ given by

$$
\Gamma(x):=\{y \in \Omega:|x-y|<2 \operatorname{dist}(y, \partial \Omega)\} .
$$

Also, for any open subset $E$ of $\partial \Omega$, the trace $\left.w\right|_{E}$ is understood in the nontangential sense, i.e.,

$$
\left.w\right|_{E}(x):=\lim _{\substack{y \rightarrow x \\ y \in \Gamma(x)}} w(y), \quad \text { for a.e. } \quad x \in E .
$$

Of course, the above considerations have natural counterparts to vector-valued functions.

We now turn our attention to the algebraic formalism associated with the Stokes system. Consider the following family of coefficients (with $\delta_{j k}$ denoting the Kronecker symbol):

$$
a_{j k}^{\alpha \beta}(r):=\delta_{j k} \delta_{\alpha \beta}+r \delta_{j \beta} \delta_{k \alpha}, \quad 1 \leq j, k, \alpha, \beta \leq n,
$$

indexed by the parameter $r \in \mathbb{R}$ and, adopting the summation convention over repeated indices, introduce the differential operator $L_{r}$ acting on $\vec{u}=\left(u_{\beta}\right)_{1 \leq \beta \leq n}$ by

$$
\left(L_{r} \vec{u}\right)_{\alpha}:=\partial_{j}\left(a_{j k}^{\alpha \beta}(r) \partial_{k} u_{\beta}\right)=\Delta u_{\alpha}+r \partial_{\alpha}(\operatorname{div} \vec{u}), \quad 1 \leq \alpha \leq n .
$$

Then, for each $r \in \mathbb{R}$, the Stokes system in a domain $\Omega \subset \mathbb{R}^{n}$ can be written as

$$
L_{r} \vec{u}=\nabla \pi, \quad \operatorname{div} \vec{u}=0 \quad \text { in } \Omega,
$$

where $\pi$ is a scalar-valued function in $\Omega$. 
Let $\vec{\nu}=\left(\nu_{1}, \ldots, \nu_{n}\right)$ denote the outward unit normal to $\partial \Omega$. Corresponding to each representation of the Stokes system (2.12) with $L_{r}$ as in (2.11), we shall associate the following co-normal derivative:

$$
\begin{aligned}
\partial_{\nu}^{r}(\vec{u}, \pi) & :=\left(\nu_{j} a_{j k}^{\alpha \beta}(r) \partial_{k} u_{\beta}-\nu_{\alpha} \pi\right)_{1 \leq \alpha \leq n} \\
& =\left[(\nabla \vec{u})^{\top}+r(\nabla \vec{u})\right] \vec{\nu}-\pi \vec{\nu} \quad \text { on } \partial \Omega,
\end{aligned}
$$

where $\nabla \vec{u}$ is the Jacobian matrix of $\vec{u}$ and $T$ indicates transposition. Then, introducing the bilinear form

$$
A_{r}(\xi, \zeta):=a_{j k}^{\alpha \beta}(r) \xi_{j}^{\alpha} \zeta_{k}^{\beta}, \quad \forall \xi, \zeta n \times n \text { matrices },
$$

we have the following useful integration by parts formulas (where $\langle\cdot, \cdot\rangle$ stands for the standard inner product in $\mathbb{R}^{n}$ ):

$$
\int_{\Omega}\left\langle L_{r} \vec{u}-\nabla \pi, \vec{w}\right\rangle d x=\int_{\partial \Omega}\left\langle\partial_{\nu}^{r}(\vec{u}, \pi), \vec{w}\right\rangle d \sigma-\int_{\Omega}\left[A_{r}(\nabla \vec{u}, \nabla \vec{w})-\pi(\operatorname{div} \vec{w})\right] d x
$$

and

$$
\begin{aligned}
\int_{\Omega}\left\langle L_{r} \vec{u}-\nabla \pi, \vec{w}\right\rangle d x-\int_{\Omega}\left\langle L_{r} \vec{w}-\nabla \rho, \vec{u}\right\rangle d x \\
\quad=\int_{\partial \Omega}\left\langle\partial_{\nu}^{r}(\vec{u}, \pi), \vec{w}\right\rangle d \sigma-\int_{\partial \Omega}\left\langle\partial_{\nu}^{r}(\vec{w}, \rho), \vec{u}\right\rangle d \sigma+\int_{\Omega} \pi(\operatorname{div} \vec{w}) d x-\int_{\Omega} \rho(\operatorname{div} \vec{u}) d x .
\end{aligned}
$$

For future reference, let us also record here that

$$
A_{r}(\zeta, \zeta) \geq C_{r}\left\{\begin{array}{l}
\left|\zeta+\zeta^{\top}\right|^{2} \text { if } r \in(-1,1], \\
|\zeta|^{2} \text { if }|r|<1,
\end{array}\right.
$$

uniformly in the $n \times n$ matrix $\zeta$.

We continue by reviewing background material about the layer potentials associated with the Stokes system in a Lipschitz domain $\Omega \subset \mathbb{R}^{n}$. Let $\omega_{n-1}$ denote the surface measure of $S^{n-1}$, the unit sphere in $\mathbb{R}^{n}$, and let $E(x)=\left(E_{j k}(x)\right)_{1 \leq j, k \leq n}$ be the Kelvin matrix of fundamental solutions for the Stokes system, where

$$
E_{j k}(x):=-\frac{1}{2 \omega_{n-1}}\left(\frac{1}{n-2} \frac{\delta_{j k}}{|x|^{n-2}}+\frac{x_{j} x_{k}}{|x|^{n}}\right), \quad x \in \mathbb{R}^{n} \backslash\{0\}, \quad n \geq 3 .
$$

A direct calculation shows that, for $x \in \mathbb{R}^{n} \backslash\{0\}$,

$$
\partial_{k} E_{j k}(x)=0 \text { for } 1 \leq j \leq n,
$$

where, as before, summation is performed over repeated indices.

Let us also introduce a pressure vector $\vec{q}(x)$ given by

$$
\vec{q}(x)=\left(q_{j}(x)\right)_{1 \leq j \leq n}:=-\frac{1}{\omega_{n-1}} \frac{x}{|x|^{n}}, \quad x \in \mathbb{R}^{n} \backslash\{0\} .
$$


Now, define the single layer potential operators $\mathcal{S}$ and $S$ by setting, respectively,

$$
\begin{aligned}
\mathcal{S} \vec{f}(x):=\int_{\partial \Omega} E(x-y) \vec{f}(y) d \sigma(y), & x \in \Omega, \\
S \vec{f}(x):=\int_{\partial \Omega} E(x-y) \vec{f}(y) d \sigma(y), & x \in \partial \Omega .
\end{aligned}
$$

In particular,

$$
\left.\mathcal{S} \vec{f}\right|_{\partial \Omega}=S \vec{f} \quad \text { on } \partial \Omega
$$

For future purposes we note here that, as a consequence of (2.19) and the Divergence Theorem,

$$
\mathcal{S}(\vec{\nu})=0 \text { in } \Omega \Longrightarrow S(\vec{\nu})=0 \text { on } \partial \Omega .
$$

Let us also define a corresponding potential for the pressure by

$$
\mathcal{Q} \vec{f}(x):=\int_{\partial \Omega}\langle\vec{q}(x-y), \vec{f}(y)\rangle d \sigma(y), \quad x \in \Omega,
$$

so that

$$
\mathcal{Q}(\vec{\nu})(x)=\frac{1}{\omega_{n-1}} \int_{\partial \Omega} \frac{\langle\vec{\nu}(y), y-x\rangle}{|x-y|^{n}} d \sigma(y)=1 \quad \text { for } \quad x \in \Omega .
$$

Furthermore,

$$
\Delta \mathcal{S} \vec{f}-\nabla \mathcal{Q} \vec{f}=0 \quad \text { and } \quad \operatorname{div} \mathcal{S} \vec{f}=0 \quad \text { in } \quad \Omega .
$$

These layer potentials also satisfy the following trace formulas:

$$
\begin{aligned}
\left.\partial_{j} \mathcal{S} \vec{f}\right|_{\partial \Omega}(x) & =-\frac{1}{2} \nu_{j}(x) \vec{f}_{\text {tan }}(x)+\text { p.v. } \int_{\partial \Omega}\left(\partial_{j} E\right)(x-y) \vec{f}(y) d \sigma(y), \\
\left.\mathcal{Q} \vec{f}\right|_{\partial \Omega}(x) & =\frac{1}{2}\langle\nu(x), \vec{f}(x)\rangle+\text { p.v. } \int_{\partial \Omega}\langle\vec{q}(x-y), \vec{f}(y)\rangle d \sigma(y)=: Q \vec{f}(x),
\end{aligned}
$$

at a.e. $x \in \partial \Omega$, where the subscript tan indicates the tangential component. It follows from (2.28) and (2.29) that

$$
\left.\partial_{\nu}^{r}(\mathcal{S} \vec{f}, \mathcal{Q} \vec{f})\right|_{\partial \Omega}(x)=\left(-\frac{1}{2} I+K_{r}\right) \vec{f}(x), \quad \text { a.e. } \quad x \in \partial \Omega,
$$

where, with p.v. denoting principal value (in the usual sense of excluding small, concentric balls shrinking to the singularity),

$$
\begin{aligned}
& K_{r} \vec{f}(x)=\text { p.v. } \int_{\partial \Omega}(1-r) \frac{\langle\nu(x), x-y\rangle \vec{f}(y)+\nu(x)\langle x-y, \vec{f}(y)\rangle-(x-y)\langle\nu(x), \vec{f}(y)\rangle}{2 \omega_{n-1}|x-y|^{n}} d \sigma_{y} \\
& \quad+\text { p.v. } \int_{\partial \Omega}(1+r) \frac{n(x-y)\langle\nu(x), x-y\rangle\langle x-y, \vec{f}(y)\rangle}{2 \omega_{n-1}|x-y|^{n+2}} d \sigma_{y}, \quad x \in \partial \Omega .
\end{aligned}
$$

We conclude this section by pointing out that, thanks to the results in $[5]$ and the techniques in [8], we have

$$
\|M(\nabla \mathcal{S} \vec{f})\|_{L^{2}(\partial \Omega)}+\|M(\mathcal{Q} \vec{f})\|_{L^{2}(\partial \Omega)} \leq C\|\vec{f}\|_{L^{2}(\partial \Omega)}
$$


and the operators

$$
\nabla_{t a n} S: L^{2}(\partial \Omega) \longrightarrow L^{2}(\partial \Omega), \quad K_{r}: L^{2}(\partial \Omega) \longrightarrow L^{2}(\partial \Omega)
$$

are well-defined and bounded. For more detailed discussions on these topics see, e.g., [9], 21].

\section{REDUCTION OF THE MIXED PROBLEM TO A BOUNDARY EQUATION}

Let $\Omega \subseteq \mathbb{R}^{n}, n \geq 3$, be a graph Lipschitz creased domain. With $r \in(-1,1]$ fixed, consider the following boundary value problem:

$$
\left\{\begin{array}{l}
\Delta \vec{u}=\nabla \pi \text { and } \operatorname{div} \vec{u}=0 \text { in } \Omega, \\
\left.\vec{u}\right|_{D}=\vec{f}_{D} \in \dot{L}_{1}^{2}(D) \text { and }\left.\partial_{\nu}^{r}(\vec{u}, \pi)\right|_{N}=\vec{f}_{N} \in L^{2}(N), \\
M(\nabla \vec{u}), M(\pi) \in L^{2}(\partial \Omega) .
\end{array}\right.
$$

For $r \in(-1,1]$, define the operator

$$
T_{r}: L^{2}(\partial \Omega) \longrightarrow \dot{L}_{1}^{2}(D) \oplus L^{2}(N), \quad T_{r} \vec{f}:=\left(\left.(S \vec{f})\right|_{D},\left.\left(\left(-\frac{1}{2} I+K_{r}\right) \vec{f}\right)\right|_{N}\right) .
$$

We now recall two results proved in [21, where some of the work in [7] and [9] has been refined.

Lemma 3.1. Let $\Omega \subseteq \mathbb{R}^{n}, n \geq 3$, be a graph Lipschitz domain. Then the operator

$$
S: L^{2}(\partial \Omega) \longrightarrow \dot{L}_{1}^{2}(\partial \Omega)
$$

is an isomorphism.

Lemma 3.2. Let $\Omega \subseteq \mathbb{R}^{n}, n \geq 3$, be a graph Lipschitz domain. If $\vec{u}$ and $\pi$ satisfy

$$
\Delta \vec{u}=\nabla \pi \quad \text { and } \quad \operatorname{div} \vec{u}=0 \text { in } \Omega, \quad M(\nabla \vec{u}), M(\pi) \in L^{2}(\partial \Omega),
$$

then there exists $\vec{f} \in L^{2}(\partial \Omega)$ and $\vec{c} \in \mathbb{R}^{n}$ such that $\vec{u}=\mathcal{S} \vec{f}+\vec{c}$ in $\Omega$ and $\pi=\mathcal{Q} \vec{f}$ in $\Omega$.

With these results in mind, we can prove the following theorem.

Theorem 3.3. Let $\Omega$ be a graph Lipschitz creased domain. Then for $r \in(-1,1]$ fixed, the following are equivalent:

(1) the mixed boundary value problem (3.1) is well-posed;

(2) the operator $T_{r}$ defined in (3.2) is an isomorphism.

Proof. Fix $r \in(-1,1]$. Assume (3.1) is well-posed, and let $\vec{f}_{D} \in \dot{L}_{1}^{2}(D)$ and $\vec{f}_{N} \in$ $L^{2}(N)$. Then there exist $\vec{u}$ and $\pi$ that satisfy (3.1). From Lemma 3.2 there exist $\vec{f} \in L^{2}(\partial \Omega)$ and $\vec{c} \in \mathbb{R}^{n}$ such that $\vec{u}=\mathcal{S} \vec{f}+\vec{c}$ and $\pi=\mathcal{Q} \vec{f}$. Then it follows that $T_{r} \vec{f}=\left(\left.\vec{u}\right|_{D},\left.\partial_{\nu}^{r}(\vec{u}, \pi)\right|_{N}\right)=\left(\vec{f}_{D}, \vec{f}_{N}\right)$, and so $T_{r}$ is surjective. To check its injectivity, suppose that $\vec{f} \in L^{2}(\partial \Omega)$ satisfies $T_{r} \vec{f}=0$. Then $\vec{u}=\mathcal{S} \vec{f}$ and $\pi=\mathcal{Q} \vec{f}$ solve the homogeneous version of (3.1). See (2.27) and (2.32). Hence, from uniqueness, $\vec{u}$ must be constant in $\Omega$. Consequently, $S \vec{f}=\left.\vec{u}\right|_{\partial \Omega}=0$ in $\dot{L}_{1}^{2}(\partial \Omega)$, which forces $\vec{f}=0$ by Lemma 3.1. This shows that $T_{r}$ is injective. Thus $T_{r}$ is an isomorphism, as desired.

Next, assume that $T_{r}$ is an isomorphism. Since $T_{r}$ is surjective, for any $\vec{f}_{D} \in$ $\dot{L}_{1}^{2}(D)$ and $\vec{f}_{N} \in L^{2}(N)$, there exists $\vec{f} \in L^{2}(\partial \Omega)$ such that $T_{r} \vec{f}=\left(\vec{f}_{D}, \vec{f}_{N}\right)$. Then 
$\vec{u}:=\mathcal{S} \vec{f}$ and $\pi:=\mathcal{Q} \vec{f}$ in $\Omega$ solve (3.1) and, since $T_{r}$ is an isomorphism, they also satisfy the estimate

$$
\begin{aligned}
\|M(\nabla \vec{u})\|_{L^{2}(\partial \Omega)}+\|M(\pi)\|_{L^{2}(\partial \Omega)} & \leq C\|\vec{f}\|_{L^{2}(\partial \Omega)} \leq C\left\|T_{r} \vec{f}\right\|_{\dot{L}_{1}^{2}(D) \oplus L^{2}(N)} \\
& =C\left\|\vec{f}_{D}\right\|_{\dot{L}_{1}^{2}(D)}+C\left\|\vec{f}_{N}\right\|_{L^{2}(N)} .
\end{aligned}
$$

To prove uniqueness, assume $\vec{u}$ and $\pi$ solve the homogeneous version of (3.1). Then by Lemma 3.2, $\vec{u}=\mathcal{S} \vec{f}+\vec{c}$ and $\pi=\mathcal{Q} \vec{f}$ for some $\vec{f} \in L^{2}(\partial \Omega)$ and $\vec{c} \in \mathbb{R}^{n}$. It follows that $T_{r} \vec{f}=0$, and since $T_{r}$ is injective, $\vec{f}=0$. Then $\vec{u}=\vec{c}$ and $\pi=0$, as desired.

\section{Rellich estimates}

The main result in this section is the theorem below, which contains the key technical estimate of the paper. In the case when either $D$ or $N$ is empty, our Rellich-type estimate below reduces to those proved in [7] and [9]. For example, if $D=\emptyset$, our Rellich estimate (on graphs) implies

$$
\int_{\partial \Omega}\left[|\nabla \vec{u}|^{2}+\pi^{2}\right] d \sigma \leq C \int_{\partial \Omega}\left|\left[\nabla \vec{u}+(\nabla \vec{u})^{\top}\right] \vec{\nu}-\pi \vec{\nu}\right|^{2} d \sigma
$$

which has been proved in [7] using a so-called boundary Korn estimate. However, it should be noted that the more general case $(D \neq \emptyset)$ treated here is more delicate, in that one cannot use the boundary Korn inequality from [7] directly in the present setting. The reason is that one needs to work with algebraic identities, instead of estimates, in order to facilitate further cancellations. Hence, the challenge is to find such suitable Rellich-type identities which, in some sense, have a built-in boundary Korn estimate.

Theorem 4.1. Let $\Omega$ be a Lipschitz creased domain in $\mathbb{R}^{n}, n \geq 2$. Let $\partial \Omega=N \cup D$ with $N \cap D=\emptyset$. For $r \in(-1,1]$ fixed, there exists $C>0$ such that if $\vec{u}$ and $\pi$ satisfy

$$
L_{r} \vec{u}=\nabla \pi \quad \text { and } \quad \operatorname{div} \vec{u}=0 \quad \text { in } \Omega, \quad M(\nabla \vec{u}), M(\pi) \in L^{2}(\partial \Omega),
$$

then

$$
\int_{\partial \Omega}\left[|\nabla \vec{u}|^{2}+\pi^{2}\right] d \sigma \leq C \int_{N}\left|\partial_{\nu}^{r}(\vec{u}, \pi)\right|^{2} d \sigma+C \int_{D}\left|\nabla_{t a n} \vec{u}\right|^{2} d \sigma+C \int_{\Omega}\left[|\nabla \vec{u}|^{2}+\pi^{2}\right] d x .
$$

Proof. First, for any $\vec{u}$ and $\pi$ as in (4.2) and any $\vec{h} \in C^{\infty}\left(\mathbb{R}^{n}\right)$, the Gauss divergence theorem leads to the following Rellich-type identities:

$$
\begin{aligned}
\int_{\partial \Omega} A_{r}(\nabla \vec{u}, \nabla \vec{u})\langle\vec{h}, \vec{\nu}\rangle d \sigma= & 2 \int_{\partial \Omega}\left\langle\partial_{\nu}^{r}(\vec{u}, \pi), \nabla_{h} \vec{u}\right\rangle d \sigma+\int_{\Omega}(\operatorname{div} \vec{h}) A_{r}(\nabla \vec{u}, \nabla \vec{u}) d x \\
& +2 \int_{\Omega}\left[\pi\left(\partial_{i} u_{k}\right)\left(\partial_{k} h_{i}\right)-\left(\partial_{i} u_{k}\right)\left(\partial_{j} u_{k}+r \partial_{k} u_{j}\right)\left(\partial_{j} h_{i}\right)\right] d x \\
(4.4) \quad & 2 \int_{\partial \Omega}\left\langle\partial_{\nu}^{r}(\vec{u}, \pi), \nabla_{h} \vec{u}\right\rangle d \sigma+\int_{\Omega} \mathcal{O}_{h} d x
\end{aligned}
$$


and

$$
\begin{aligned}
\int_{\partial \Omega} \pi^{2}\langle\vec{h}, \vec{\nu}\rangle d \sigma= & -2 \int_{\partial \Omega}\left\langle\partial_{\nu}^{-1}(\vec{u}, \pi),(\nabla \vec{u}) \vec{h}\right\rangle d \sigma+\int_{\Omega}(\operatorname{div} \vec{h})(\pi)^{2} d x \\
& +2 \int_{\Omega}\left[\left(\partial_{k} u_{i}\right)\left(\partial_{j} h_{i}\right)\left(\partial_{j} u_{k}-\partial_{k} u_{j}\right)-\left(\partial_{j} h_{i}\right)\left(\partial_{j} u_{i}\right) \pi\right] d x \\
= & -2 \int_{\partial \Omega}\left\langle\partial_{\nu}^{-1}(\vec{u}, \pi),(\nabla \vec{u}) \vec{h}\right\rangle d \sigma+\int_{\Omega} \mathcal{O}_{h} d x .
\end{aligned}
$$

Here and elsewhere, $\vec{h}=\left(h_{k}\right)_{1 \leq k \leq n}, \vec{u}=\left(u_{j}\right)_{1 \leq j \leq n}$, and we have used the notation $\nabla_{h} \vec{u}:=\left(h_{k} \partial_{k} u_{j}\right)_{1 \leq j \leq n}$. From here on, let us also use $\mathcal{O}_{h}$ to denote any function in $\Omega$ such that

$$
\mathcal{O}_{h} \leq C\left(|\nabla \vec{u}|^{2}+\pi^{2}\right)|\nabla \vec{h}|
$$

for some finite, purely dimensional constant $C>0$. If we choose $\vec{h}$ such that $\langle\vec{h}, \vec{\nu}\rangle>\delta$ almost everywhere on $\partial \Omega$ (which is possible, given that $\partial \Omega$ is Lipschitz), then using Cauchy's inequality in (4.5) allows us to conclude that

$$
\int_{\partial \Omega} \pi^{2} d \sigma \leq C \int_{\partial \Omega}|\nabla \vec{u}|^{2} d \sigma+C \int_{\Omega}\left[|\nabla \vec{u}|^{2}+\pi^{2}\right] d x .
$$

Next, define the matrix valued function

$$
\nabla_{\tau}^{h} \vec{u}:=\left(h_{i} \partial_{\tau_{j i}} u_{k}\right)_{j, k} .
$$

Then the following identity holds:

$$
\left\langle\partial_{\nu}^{r}(\vec{u}, \pi), \nabla_{h} \vec{u}\right\rangle-A_{r}(\nabla \vec{u}, \nabla \vec{u})\langle\vec{h}, \vec{\nu}\rangle=A_{r}\left(\nabla \vec{u}, \nabla_{\tau}^{h} \vec{u}\right)-\pi\langle(\nabla \vec{u}) \vec{\nu}, h\rangle .
$$

Notice that since $\operatorname{div} \vec{u}=0$ in $\Omega$, we have

$$
(\nabla \vec{u}) \vec{\nu}=\left(\nu_{k} \partial_{j} u_{k}\right)_{j}=\left(\partial_{\tau_{k j}} u_{k}\right)_{j},
$$

and so both terms on the right-hand side of (4.9) include tangential derivatives. Now, subtracting the term $2 \int_{D} A_{r}(\nabla \vec{u}, \nabla \vec{u})\langle\vec{h}, \vec{\nu}\rangle d \sigma$ from both sides of (4.4) and using (4.9) gives

$$
\begin{aligned}
\int_{N} A_{r} & (\nabla \vec{u}, \nabla \vec{u})\langle\vec{h}, \vec{\nu}\rangle d \sigma-\int_{D} A_{r}(\nabla \vec{u}, \nabla \vec{u})\langle\vec{h}, \vec{\nu}\rangle d \sigma \\
= & \int_{N}\left\langle\partial_{\nu}^{r}(\vec{u}, \pi), \nabla_{h} \vec{u}\right\rangle d \sigma+\int_{D}\left[A_{r}\left(\nabla \vec{u}, \nabla_{\tau}^{h} \vec{u}\right)-\pi\langle(\nabla \vec{u}) \vec{\nu}, h\rangle\right] d \sigma+\int_{\Omega} \mathcal{O}_{h} d x .
\end{aligned}
$$

At this stage, we bring in the condition that $\Omega$ is a creased domain, so we can choose a vector field $\vec{h} \in C_{0}^{\infty}\left(\mathbb{R}^{n}\right)$ with the property that (2.2) holds for some $\delta>0$. Using (2.2) in (4.11) and Cauchy's inequality with epsilon (i.e., $a b \leq \varepsilon a^{2}+(4 \varepsilon)^{-1} b^{2}$ ) 
gives

$$
\begin{aligned}
& \int_{\partial \Omega} A_{r}(\nabla \vec{u}, \nabla \vec{u}) d \sigma \leq C_{\varepsilon} \int_{N}\left|\partial_{\nu}^{r}(\vec{u}, \pi)\right|^{2} d \sigma+C_{\varepsilon} \int_{D}\left|\nabla_{\tan } \vec{u}\right|^{2} d \sigma \\
& \quad+\varepsilon \int_{\partial \Omega}|\nabla \vec{u}|^{2} d \sigma+\varepsilon \int_{D} \pi^{2} d \sigma+C \int_{\Omega}\left[|\nabla \vec{u}|^{2}+\pi^{2}\right] d x .
\end{aligned}
$$

If $|r|<1$, then $|\nabla \vec{u}|^{2} \leq C_{r} A_{r}(\nabla \vec{u}, \nabla \vec{u})$ by (2.17), and so (4.3) follows from (4.12) and (4.7) after choosing $\varepsilon$ small enough. Now, consider the case when $r=1$. Subtracting one-fourth of (4.5) from (4.11) with $r=0$ leads to

$$
\begin{aligned}
\int_{N} & {\left[|\nabla \vec{u}|^{2}-\frac{1}{4} \pi^{2}\right]\langle\vec{h}, \vec{\nu}\rangle d \sigma-\int_{D}\left[|\nabla \vec{u}|^{2}+\frac{1}{4} \pi^{2}\right]\langle\vec{h}, \vec{\nu}\rangle d \sigma } \\
& =\int_{N}\left\langle\partial_{\nu}^{0}(\vec{u}, \pi), \nabla_{h} \vec{u}\right\rangle d \sigma+\int_{D}\left[A_{0}\left(\nabla \vec{u}, \nabla_{\tau}^{h} \vec{u}\right)-\pi\langle(\nabla \vec{u}) \vec{\nu}, h\rangle\right] d \sigma \\
3) \quad & +\frac{1}{2} \int_{\partial \Omega}\left\langle\partial_{\nu}^{-1}(\vec{u}, \pi),(\nabla \vec{u}) \vec{h}\right\rangle d \sigma+\int_{\Omega} \mathcal{O}_{h} d x \\
= & \frac{1}{2} \int_{N}\left\langle\partial_{\nu}^{1}(\vec{u}, \pi), \nabla_{h} \vec{u}\right\rangle d \sigma+\int_{D}\left[A_{0}\left(\nabla \vec{u}, \nabla_{\tau}^{h} \vec{u}\right)-\pi\langle(\nabla \vec{u}) \vec{\nu}, h\rangle\right] d \sigma \\
& -\frac{1}{2} \int_{D}\left\langle\partial_{\nu}^{-1}(\vec{u}, \pi), \nabla_{h} \vec{u}\right\rangle d \sigma+\frac{1}{2} \int_{\partial \Omega}\left\langle\partial_{\nu}^{-1}(\vec{u}, \pi),\left(\nabla \vec{u}+\nabla \vec{u}^{\top}\right) \vec{h}\right\rangle d \sigma+\int_{\Omega} \mathcal{O}_{h} d x .
\end{aligned}
$$

Then since

$$
\left\langle\partial_{\nu}^{-1}(\vec{u}, \pi), \nabla_{h} \vec{u}\right\rangle=\left\langle\left(\nabla \vec{u}+\nabla \vec{u}^{\top}\right) \vec{\nu}, \nabla_{h} \vec{u}\right\rangle-\left\langle(\nabla \vec{u}) \vec{\nu}, 2 \nabla_{h} \vec{u}+\pi \vec{h}\right\rangle,
$$

we further obtain

$$
\begin{aligned}
& \int_{N}|\nabla \vec{u}|^{2}\langle\vec{h}, \vec{\nu}\rangle d \sigma-\int_{D}\left[|\nabla \vec{u}|^{2}+\frac{1}{4} \pi^{2}\right]\langle\vec{h}, \vec{\nu}\rangle d \sigma \\
& \quad=\frac{1}{2} \int_{N}\left[\left\langle\partial_{\nu}^{1}(\vec{u}, \pi), \nabla_{h} \vec{u}\right\rangle+\frac{1}{2} \pi^{2}\langle\vec{h}, \vec{\nu}\rangle\right] d \sigma \\
& \quad+\int_{D}\left[A_{0}\left(\nabla \vec{u}, \nabla_{\tau}^{h} \vec{u}\right)-\frac{1}{2}\left\langle\left(\nabla \vec{u}+\nabla \vec{u}^{\top}\right) \vec{\nu}, \nabla_{h} \vec{u}\right\rangle\right] d \sigma \\
& \quad+\int_{D}\left\langle(\nabla \vec{u}) \vec{\nu}, \nabla_{h} \vec{u}-\frac{1}{2} \pi \vec{h}\right\rangle d \sigma+\frac{1}{2} \int_{\partial \Omega}\left\langle\partial_{\nu}^{-1}(\vec{u}, \pi),\left(\nabla \vec{u}+\nabla \vec{u}^{\top}\right) \vec{h}\right\rangle d \sigma+\int_{\Omega} \mathcal{O}_{h} d x
\end{aligned}
$$

Also, since $\pi=\left\langle\left(\nabla \vec{u}+\nabla \vec{u}^{\top}\right) \vec{\nu}-\partial_{\nu}^{1}(\vec{u}, \pi), \vec{\nu}\right\rangle$, we can estimate

$$
\pi^{2} \leq C\left|\nabla \vec{u}+\nabla \vec{u}^{\top}\right|^{2}+C\left|\partial_{\nu}^{1}(\vec{u}, \pi)\right|^{2} .
$$


Let $\vec{h}$ be as in (2.2). Then applying Cauchy's inequality with epsilon and (4.16) to (4.15) gives

$$
\begin{aligned}
& \int_{\partial \Omega}|\nabla \vec{u}|^{2} d \sigma+\frac{1}{4} \int_{D} \pi^{2} d \sigma \\
& \leq C_{\varepsilon} \int_{N}\left|\partial_{\nu}^{1}(\vec{u}, \pi)\right|^{2} d \sigma+C_{\varepsilon} \int_{D}\left|\nabla_{t a n} \vec{u}\right|^{2} d \sigma+C_{\varepsilon} \int_{\partial \Omega}\left|\nabla \vec{u}+\nabla \vec{u}^{\top}\right|^{2} d \sigma \\
& \quad+\varepsilon \int_{\partial \Omega}|\nabla \vec{u}|^{2} d \sigma+\varepsilon \int_{D} \pi^{2} d \sigma+C \int_{\Omega}\left[|\nabla \vec{u}|^{2}+\pi^{2}\right] d x .
\end{aligned}
$$

Combining (4.17) and (4.12) with $r=1$, as well as (4.7), allows us to derive (4.3) by choosing appropriately small values of $\varepsilon$.

Corollary 4.2. Let $\Omega$ be a graph Lipschitz creased domain in $\mathbb{R}^{n}, n \geq 2$. Let $\partial \Omega=N \cup D$ with $N \cap D=\emptyset$. For $r \in(-1,1]$ fixed, there exists $C>0$ such that if $\vec{u}$ and $\pi$ satisfy (4.2), then

$$
\int_{\partial \Omega}\left[|\nabla \vec{u}|^{2}+\pi^{2}\right] d \sigma \leq C \int_{N}\left|\partial_{\nu}^{r}(\vec{u}, \pi)\right|^{2} d \sigma+C \int_{D}\left|\nabla_{\tan } \vec{u}\right|^{2} d \sigma .
$$

Proof. The proof follows in the same manner as the proof of Theorem 4.1, except since $\Omega$ is a graph domain, we can always choose $\vec{h}$ to be a constant vector field, and so any terms involving derivatives of $\vec{h}$ vanish.

\section{The CASE OF A CREASED GRAPH Lipschitz DOMAIN}

In this section we formulate and solve the mixed boundary value problem for the Stokes system in a creased graph Lipschitz domain in $\mathbb{R}^{n}, n \geq 3$. The starting point is the following.

Proposition 5.1. Let $\Omega$ be a graph Lipschitz creased domain. For $r \in(-1,1]$ fixed, there exists $C>0$ such that

$$
\|\vec{f}\|_{L^{2}(\partial \Omega)} \leq C\left\|T_{r} \vec{f}\right\|_{\dot{L}_{1}^{2}(D) \oplus L^{2}(N)}, \quad \forall \vec{f} \in L^{2}(\partial \Omega) .
$$

Proof. From Lemma 3.1, there exists $C>0$ such that

$$
\|\vec{f}\|_{L^{2}(\partial \Omega)} \leq C\left\|\nabla_{\tan } S \vec{f}\right\|_{L^{2}(\partial \Omega)}, \quad \forall \vec{f} \in L^{2}(\partial \Omega) .
$$

For $\vec{f} \in L^{2}(\partial \Omega)$ arbitrary and fixed, set $\vec{u}:=\mathcal{S} \vec{f}$ and $\pi:=\mathcal{Q} \vec{f}$ in $\Omega$, so that (3.4) holds. The sequence of estimates proving (5.1) then reads

$$
\begin{aligned}
\|\vec{f}\|_{L^{2}(\partial \Omega)} & \leq C\left\|\nabla_{\tan } S \vec{f}\right\|_{L^{2}(\partial \Omega)} \leq C\|\nabla \vec{u}\|_{L^{2}(\partial \Omega)} \\
& \leq C\left\|\left(\left.\vec{u}\right|_{D},\left.\partial_{\nu}^{r}(\vec{u}, \pi)\right|_{N}\right)\right\|_{\dot{L}_{1}^{2}(D) \oplus L^{2}(N)}=C\left\|T_{r} \vec{f}\right\|_{\dot{L}_{1}^{2}(D) \oplus L^{2}(N)} .
\end{aligned}
$$

Above, we have used Lemma 3.1, (2.23), Corollary 4.2, and (3.2).

The following refines Proposition 5.1 .

Theorem 5.2. Let $\Omega$ be a graph Lipschitz creased domain. Then for $r \in(-1,1]$, the operator $T_{r}$ defined in (3.2) is an isomorphism. 
In order to prove this theorem, we will next consider a similar mixed boundary value problem for the Lamé system. For each $\mu, \lambda, r \in \mathbb{R}$, let

$$
\tilde{a}_{j k}^{\alpha \beta}(r):=\mu \delta_{j k} \delta_{\alpha \beta}+r \delta_{j \beta} \delta_{k \alpha}+(\mu+\lambda-r) \delta_{j \alpha} \delta_{k \beta}, \quad 1 \leq j, k, \alpha, \beta \leq n,
$$

and define

$$
\left(\tilde{L}_{r} \vec{u}\right)_{\alpha}:=\partial_{j}\left(\tilde{a}_{j k}^{\alpha \beta}(r) \partial_{k} u_{\beta}\right)=\mu \Delta u_{\alpha}+(\mu+\lambda) \partial_{\alpha}(\operatorname{div} \vec{u}) .
$$

Let us also consider the conormal derivative associated with the writing (5.4)(5.5), namely

$$
\begin{aligned}
\partial_{\nu}^{r} \vec{u} & :=\left(\nu_{j} \tilde{a}_{j k}^{\alpha \beta}(r) \partial_{k} u_{\beta}\right)_{1 \leq \alpha \leq n} \\
& =\left(\mu \nabla \vec{u}^{\top}+r \nabla \vec{u}\right) \vec{\nu}+(\mu+\lambda-r)(\operatorname{div} \vec{u}) \vec{\nu} .
\end{aligned}
$$

Assume next that

$$
\mu>0, \quad \lambda \geq-\frac{2 \mu}{n}, \quad \text { and } \quad r \in(-\mu, \mu],
$$

and consider the boundary value problem

$$
\left\{\begin{array}{l}
\mu \Delta \vec{u}+(\mu+\lambda) \nabla(\operatorname{div} \vec{u})=0 \text { in } \Omega, \\
\left.\vec{u}\right|_{D}=\vec{f}_{D} \in \dot{L}_{1}^{2}(D), \\
\left.\partial_{\nu}^{r} \vec{u}\right|_{N}=\vec{f}_{N} \in L^{2}(N), \\
M(\nabla \vec{u}) \in L^{2}(\partial \Omega) .
\end{array}\right.
$$

Recall the (matrix-valued) fundamental solution for the Lamé system, $E^{\mu, \lambda}:=$ $\left(E_{j k}^{\mu, \lambda}\right)_{1 \leq j, k \leq n}$, given by

$$
\begin{aligned}
& E_{j k}^{\mu, \lambda}(x):=-\frac{1}{2 \omega_{n-1}}\left(\frac{3 \mu+\lambda}{\mu(2 \mu+\lambda)} \frac{1}{n-2} \frac{\delta_{j k}}{|x|^{n-2}}+\frac{\mu+\lambda}{\mu(2 \mu+\lambda)} \frac{x_{j} x_{k}}{|x|^{n}}\right), \\
& x \in \mathbb{R}^{n} \backslash\{0\}, \quad n \geq 3 .
\end{aligned}
$$

Then, in parallel to (2.21)-(2.22), we can define a single layer potential operator $\mathcal{S}_{\mu, \lambda}$ for the Lamé system as well as its boundary version $S_{\mu, \lambda}$. These operators will satisfy a trace formula much like (2.23) and a jump-formula similar to (2.30). More precisely, we have

$$
\left.\partial_{\nu}^{r}\left(\mathcal{S}_{\mu, \lambda} \vec{f}\right)\right|_{\partial \Omega}=\left(-\frac{1}{2} I+K_{\mu, \lambda, r}\right) \vec{f}
$$

where, at a.e. $x \in \partial \Omega$, we have set

$$
\begin{aligned}
K_{\mu, \lambda, r} \vec{f}(x) & :=\text { p.v. } \int_{\partial \Omega}\left[\frac{(3-r) \mu+(1-r) \lambda}{\mu(2 \mu+\lambda)}\right] \frac{\langle\vec{\nu}(x), x-y\rangle \vec{f}(y)}{2 \omega_{n-1}|x-y|^{n}} d \sigma_{y} \\
& + \text { p.v. } \int_{\partial \Omega}\left[\frac{\mu^{2}-3 r \mu+(\mu-r) \lambda}{\mu(2 \mu+\lambda)}\right] \frac{\vec{\nu}(x)\langle x-y, \vec{f}(y)\rangle-(x-y)\langle\vec{\nu}(x), \vec{f}(y)\rangle}{2 \omega_{n-1}|x-y|^{n}} d \sigma_{y} \\
& + \text { p.v. } \int_{\partial \Omega}\left[\frac{(\mu+r)(\mu+\lambda)}{\mu(2 \mu+\lambda)}\right] \frac{n(x-y)\langle\vec{\nu}(x), x-y\rangle\langle x-y, \vec{f}(y)\rangle}{2 \omega_{n-1}|x-y|^{n+2}} d \sigma_{y} .
\end{aligned}
$$


Let us also note that the analogues of (2.32)-(2.33) hold in this setting as well.

Next, in parallel to (3.2), we define an operator

$$
\begin{aligned}
& T_{\mu, \lambda, r}: L^{2}(\partial \Omega) \longrightarrow \dot{L}_{1}^{2}(D) \oplus L^{2}(N), \\
& T_{\mu, \lambda, r} \vec{f}:=\left(\left.\left(S_{\mu, \lambda} \vec{f}\right)\right|_{D},\left.\left(\left(-\frac{1}{2} I+K_{\mu, \lambda, r}\right) \vec{f}\right)\right|_{N}\right) .
\end{aligned}
$$

In a recent paper 4, R. Brown and I. Mitrea proved that the mixed boundary problem (5.8) for the Lamé system is well-posed for all $\mu, \lambda, r$ as in (5.7). Then since a result similar to Theorem 3.3 also holds for the Lamé system, it follows that

$$
T_{\mu, \lambda, r} \text { is an isomorphism for } \mu, \lambda, r \text { as in (5.7). }
$$

The reason for this discussion of the Lamé system is the following interesting connection between the layer potentials associated with the Stokes and Lamé systems.

Lemma 5.3. For each $r \in(-1,1]$, recall that $T_{r}$ is the operator associated with the Stokes problem as in (3.2) and that $T_{\mu, \lambda, r}$ is the operator associated with the Lamé system as in (5.12). Then

$$
T_{r}=\lim _{\lambda \rightarrow \infty} T_{1, \lambda, r}
$$

in the strong, operator norm sense.

Proof. This can be seen by directly comparing the corresponding kernels of the operators $T_{r}$ and $T_{1, \lambda, r}$. In particular, it follows by comparing (2.18) and (5.9) that $\lim _{\lambda \rightarrow \infty} E_{j k}^{1, \lambda}(x)=E_{j k}(x)$, and therefore $\lim _{\lambda \rightarrow \infty} S_{1, \lambda}=S$ as operators from $L^{2}(\partial \Omega)$ into $\dot{L}_{1}^{2}(\partial \Omega)$. In a similar manner, it is clear by comparing (2.31) and (5.11) that $\lim _{\lambda \rightarrow \infty} K_{1, \lambda, r}=K_{r}$ as operators on $L^{2}(\partial \Omega)$.

Having established Proposition 5.1 and Lemma 5.3. we turn our attention to the

Proof of Theorem [5.2. To get started, fix $r \in(-1,1]$ and note that $T_{r}$ in (3.2) is injective and has closed range, by Proposition 5.1. In particular, $T_{r}$ is a semiFredholm operator. Next, from (5.13), each operator $T_{1, \lambda, r}$ is Fredholm with index zero, granted that $\lambda \geq-2 / n$ (cf. (5.7)). It then follows from (5.14) and the homotopic invariance of the index that $T_{r}$ must also have index zero. Having proved this, we may then conclude that $T_{r}$ is, in fact, an isomorphism by recalling that $T_{r}$ is also injective.

Theorem 5.4. Let $\Omega$ be a graph Lipschitz creased domain in $\mathbb{R}^{n}, n \geq 3$. Then the mixed boundary value problem (3.1) is well-posed.

Proof. This is an immediate consequence of Theorem 3.3 and Theorem 5.2

\section{The CASE OF A CREASED BOUNDED LipsChitz DOMAIN}

Fix a bounded Lipschitz domain $\Omega \subset \mathbb{R}^{n}$ with connected boundary, and let $D$ be a nonempty open subset of $\partial \Omega$. More conditions will be imposed later, but, for now, we wish to recall several useful estimates valid in this setting. First, Korn's inequality gives us the fact that there exists $C=C(\partial \Omega, D)>0$ such that

$$
\int_{\Omega}\left[|\nabla \vec{u}|^{2}+|\vec{u}|^{2}\right] d x \leq C\left(\int_{\Omega}\left|\nabla \vec{u}+(\nabla \vec{u})^{\top}\right|^{2} d x+\int_{D}|u|^{2} d \sigma\right),
$$


for all vector fields $\vec{u} \in L^{2}(\Omega)$ with $\nabla \vec{u} \in L^{2}(\Omega)$. See, e.g., Corollary 5.9 in [1, or 6]. Second, under the same assumptions, the following trace inequality holds:

$$
\int_{\partial \Omega}|\vec{u}|^{2} d \sigma \leq C \int_{\Omega}\left[|\nabla \vec{u}|^{2}+|\vec{u}|^{2}\right] d x .
$$

See, e.g., [10] and [19]. Finally, we record a few versions of Poincaré's inequality on $\partial \Omega$ and $\Omega$. Specifically, there exists $C=C(\partial \Omega, D)>0$ such that

$$
\int_{\partial \Omega}|\vec{u}|^{2} d \sigma \leq C\left(\int_{\partial \Omega}\left|\nabla_{\tan } \vec{u}\right|^{2} d \sigma+\int_{D}|\vec{u}|^{2} d \sigma\right),
$$

for each vector field $\vec{u} \in L_{1}^{2}(\partial \Omega)$, and

$$
\int_{\Omega}|\vec{u}|^{2} d x \leq C\left(\int_{\Omega}|\nabla \vec{u}|^{2} d x+\int_{D}|\vec{u}|^{2} d \sigma\right),
$$

for all vector fields $\vec{u} \in L^{2}(\Omega)$ with $\nabla \vec{u} \in L^{2}(\Omega)$. Estimate (6.3) can be easily justified by locally flattening $\partial \Omega$ and then invoking well-known results in $\mathbb{R}^{n-1}$, whereas (6.4) can be found in, e.g., 1].

Moving on, let $(\vec{u}, \pi)$ be a null-solution of the Stokes system in a bounded, Lipschitz domain $\Omega \subset \mathbb{R}^{n}$, for which $M(\nabla \vec{u}), M(\pi) \in L^{2}(\partial \Omega)$. Green's formula then gives

$$
\int_{\Omega} A_{r}(\nabla \vec{u}, \nabla \vec{u}) d x=\int_{\partial \Omega}\left\langle\partial_{\nu}^{r}(\vec{u}, \pi), \vec{u}\right\rangle d \sigma .
$$

Let us also point out that, in a pointwise sense,

$$
r \in(-1,1] \Longrightarrow A_{r}(\nabla \vec{u}, \nabla \vec{u}) \geq C_{r}\left|\nabla \vec{u}+(\nabla \vec{u})^{\top}\right|^{2},
$$

for positive constants $C_{r}$ (cf. (2.17) $)$. Using (6.5), (6.6) and Korn's inequality, we may estimate

$$
\begin{aligned}
& \int_{\Omega}\left[|\nabla \vec{u}|^{2}+|u|^{2}\right] d x \leq C\left(\int_{\Omega}\left|\nabla \vec{u}+(\nabla \vec{u})^{\top}\right|^{2} d x+\int_{D}|\vec{u}|^{2} d \sigma\right) \\
& \leq C\left(\int_{\Omega} A_{r}(\nabla \vec{u}, \nabla \vec{u}) d x+\int_{D}|\vec{u}|^{2} d \sigma\right) \\
& \leq C\left(\int_{\partial \Omega}\left\langle\partial_{\nu}^{r}(\vec{u}, \pi), \vec{u}\right\rangle d \sigma+\int_{D}|\vec{u}|^{2} d \sigma\right) \\
& \leq C\left(\int_{D}\left[\left(1+\varepsilon^{-1}\right)|\vec{u}|^{2}+\varepsilon\left|\partial_{\nu}^{r}(\vec{u}, \pi)\right|^{2}\right] d \sigma+\int_{N}\left[\varepsilon|\vec{u}|^{2}+\varepsilon^{-1}\left|\partial_{\nu}^{r}(\vec{u}, \pi)\right|^{2}\right] d \sigma\right) \\
& =C_{\varepsilon}\left(\int_{D}|\vec{u}|^{2} d \sigma+\int_{N}\left|\partial_{\nu}^{r}(\vec{u}, \pi)\right|^{2} d \sigma\right)+\varepsilon\left(\int_{D}\left|\partial_{\nu}^{r}(\vec{u}, \pi)\right|^{2} d \sigma+\int_{N}|\vec{u}|^{2} d \sigma\right) .
\end{aligned}
$$

Thus, from (6.7) and (6.2),

$$
\begin{aligned}
\int_{\Omega}\left[|\nabla \vec{u}|^{2}+|u|^{2}\right] d x \leq & C_{\varepsilon}\left(\int_{D}|\vec{u}|^{2} d \sigma+\int_{N}\left|\partial_{\nu}^{r}(\vec{u}, \pi)\right|^{2} d \sigma\right) \\
& +\varepsilon \int_{D}\left|\partial_{\nu}^{r}(\vec{u}, \pi)\right|^{2} d \sigma .
\end{aligned}
$$

Next, we indicate how to estimate the pressure $\pi$ in $\Omega$, under the additional assumption that $N:=\partial \Omega \backslash \bar{D}$ is a nonempty subset of $\partial \Omega$. Specifically, we claim 
that there exists a geometrical constant $C>0$ such that

$$
\int_{\Omega} \pi^{2} d x \leq C \int_{N}\left|\partial_{\nu}^{r}(\vec{u}, \pi)\right|^{2} d \sigma+C \int_{\Omega}|\nabla \vec{u}|^{2} d x .
$$

To see this, assume there exists $\vec{w} \in L^{2}(\Omega)$ for which

$$
\left\{\begin{array}{l}
\operatorname{div} \vec{w}=\pi \text { in } \Omega, \\
\left.\vec{w}\right|_{D}=0, \\
\|\nabla \vec{w}\|_{L^{2}(\Omega)} \leq C\|\pi\|_{L^{2}(\Omega)},
\end{array}\right.
$$

where $C>0$ depends only on $\Omega$ and $D$. Note that the above conditions on $\vec{w}$ also imply

$$
\|\vec{w}\|_{L^{2}(\partial \Omega)} \leq C\|\pi\|_{L^{2}(\Omega)}
$$

by (6.2) and Poincaré's inequality (6.4). Then, from the integration by parts formula (2.15),

$$
\int_{\Omega} \pi^{2} d x=\int_{\Omega} \pi(\operatorname{div} \vec{w}) d x=\int_{\partial \Omega}\left\langle\partial_{\nu}^{r}(\vec{u}, \pi), \vec{w}\right\rangle d \sigma-\int_{\Omega} A_{r}(\nabla \vec{u}, \nabla \vec{w}) d x .
$$

Using Cauchy's inequality with epsilon along with (6.10) and (6.11) in (6.12) leads to

$$
\begin{aligned}
\int_{\Omega} \pi^{2} d x & \leq C_{\varepsilon} \int_{N}\left|\partial_{\nu}^{r}(\vec{u}, \pi)\right|^{2} d \sigma+\varepsilon \int_{N}|\vec{w}|^{2} d \sigma+C_{\varepsilon} \int_{\Omega}|\nabla \vec{u}|^{2} d x+\varepsilon \int_{\Omega}|\nabla \vec{w}|^{2} d x \\
& \leq C_{\varepsilon} \int_{N}\left|\partial_{\nu}^{r}(\vec{u}, \pi)\right|^{2} d \sigma+C_{\varepsilon} \int_{\Omega}|\nabla \vec{u}|^{2} d x+\varepsilon C \int_{\Omega} \pi^{2} d x .
\end{aligned}
$$

Then (6.9) follows by choosing $\varepsilon$ small enough.

In order to construct $\vec{w}$ as in (6.10), let $\vec{h} \in C_{0}^{\infty}\left(\mathbb{R}^{n}\right)$ be a vector field for which

$$
\left\{\begin{array}{l}
\operatorname{supp} \vec{h} \cap \partial \Omega \subseteq N, \\
\langle\vec{h}, \vec{\nu}\rangle \leq 0 \text { on } N, \\
\langle\vec{h}, \vec{\nu}\rangle \leq-\delta \text { on } N^{\prime} \subset N,
\end{array}\right.
$$

for some $\delta>0$, where $N^{\prime} \subset N$ has positive surface measure. Then, since

$$
\int_{\Omega}(\operatorname{div} \vec{h}) d x=\int_{\partial \Omega}\langle\vec{h}, \vec{\nu}\rangle d \sigma \leq \int_{N^{\prime}}\langle\vec{h}, \vec{\nu}\rangle d \sigma \leq-C \delta
$$

it follows that

$$
\lambda:=-\frac{\int_{\Omega} \pi d x}{\int_{\Omega}(\operatorname{div} \vec{h}) d x}
$$

is a well-defined real number, with the property that $|\lambda| \leq C \int_{\Omega} \pi^{2} d x$. Hence, if we now introduce $\pi^{\prime}:=\pi+\lambda \operatorname{div} \vec{h}$ in $\Omega$, we see that

$$
\int_{\Omega} \pi^{\prime} d x=0, \quad \int_{\Omega}\left(\pi^{\prime}\right)^{2} d x \leq C \int_{\Omega} \pi^{2} d x
$$


According to [2], one can then find $\vec{v} \in L^{2}(\Omega)$ such that

$$
\left\{\begin{array}{l}
\operatorname{div} \vec{v}=\pi^{\prime} \text { in } \Omega \\
\left.\vec{v}\right|_{\partial \Omega}=0 \\
\|\nabla \vec{v}\|_{L^{2}(\Omega)} \leq C\left\|\pi^{\prime}\right\|_{L^{2}(\partial \Omega)}
\end{array}\right.
$$

See also [17 for more general results of this nature. It is then easily checked that $\vec{w}:=\vec{v}-\lambda \vec{h}$ satisfies (6.10).

Having finished the proof of (6.9), we now return to the mainstream discussion. Under the additional assumption that $\Omega$ is a creased domain, a combination of (6.9) and (6.8) gives

$$
\begin{aligned}
\int_{\Omega}\left[|\nabla \vec{u}|^{2}+|\vec{u}|^{2}+|\pi|^{2}\right] d x \leq & C_{\varepsilon}\left(\int_{D}|\vec{u}|^{2} d \sigma+\int_{N}\left|\partial_{\nu}^{r}(\vec{u}, \pi)\right|^{2} d \sigma\right) \\
& +\varepsilon \int_{\partial \Omega}\left|\partial_{\nu}^{r}(\vec{u}, \pi)\right|^{2} d \sigma .
\end{aligned}
$$

Note that the last term above is $\leq \varepsilon \int_{\partial \Omega}|\nabla \vec{u}|^{2} d \sigma+\varepsilon \int_{\partial \Omega} \pi^{2} d \sigma$. Together with (4.3) and (6.2), this further yields

$$
\int_{\partial \Omega}\left[\left|\nabla_{\tan } \vec{u}\right|^{2}+|\vec{u}|^{2}+\pi^{2}\right] d \sigma \leq C \int_{N}\left|\partial_{\nu}^{r}(\vec{u}, \pi)\right|^{2} d \sigma+C \int_{D}\left[\left|\nabla_{\tan } \vec{u}\right|^{2}+|\vec{u}|^{2}\right] d \sigma .
$$

In place of Lemma 3.1 (which was used to establish (5.2)) in the graph-domain case, we shall utilize the following result.

Lemma 6.1. Let $\Omega$ be a bounded Lipschitz domain. Then there exists $C>0$ such that

$$
\|\vec{f}\|_{L^{2}(\partial \Omega)} \leq C\|S \vec{f}\|_{L_{1}^{2}(\partial \Omega)}+\|Q \vec{f}\|_{L^{2}(\partial \Omega)}, \quad \forall \vec{f} \in L^{2}(\partial \Omega),
$$

where $Q \vec{f}:=\left.\mathcal{Q} \vec{f}\right|_{\partial \Omega}$.

Proof. It has been proved in [9, 21] that the operator

$$
S: L_{\nu}^{2}(\partial \Omega) \longrightarrow L_{1, \nu}^{2}(\partial \Omega)
$$

is an isomorphism, where

$$
\begin{aligned}
L_{\nu}^{2}(\partial \Omega) & :=\left\{\vec{f} \in L^{2}(\partial \Omega): \int_{\partial \Omega}\langle\vec{f}, \vec{\nu}\rangle d \sigma=0\right\}, \\
L_{1, \nu}^{2}(\partial \Omega) & :=\left\{\vec{f} \in L_{1}^{2}(\partial \Omega): \int_{\partial \Omega}\langle\vec{f}, \vec{\nu}\rangle d \sigma=0\right\} .
\end{aligned}
$$

In particular, there exists $C>0$ such that

$$
\|\vec{g}\|_{L_{\nu}^{2}(\partial \Omega)} \leq C\|S \vec{g}\|_{L_{1, \nu}^{2}(\partial \Omega)}, \quad \forall \vec{g} \in L_{\nu}^{2}(\partial \Omega) .
$$


Going further, fix $\vec{f} \in L^{2}(\partial \Omega)$ and set $\lambda:=f_{\partial \Omega}\langle\vec{f}, \vec{\nu}\rangle d \sigma$ so that $Q(\lambda \vec{\nu})=$ $\left.\mathcal{Q}(\lambda \vec{\nu})\right|_{\partial \Omega}=\lambda$ by (2.26). Since $\vec{f}-\lambda \vec{\nu} \in L_{\nu}^{2}(\partial \Omega)$, we may write

$$
\begin{aligned}
\|\vec{f}\|_{L^{2}(\partial \Omega)} & \leq\|\lambda \vec{\nu}\|_{L^{2}(\partial \Omega)}+\|\vec{f}-\lambda \vec{\nu}\|_{L^{2}(\partial \Omega)} \\
& =\|Q(\lambda \vec{\nu})\|_{L^{2}(\partial \Omega)}+\|\vec{f}-\lambda \vec{\nu}\|_{L^{2}(\partial \Omega)} \\
& \leq\|Q \vec{f}\|_{L^{2}(\partial \Omega)}+\|Q(\vec{f}-\lambda \vec{\nu})\|_{L^{2}(\partial \Omega)}+\|\vec{f}-\lambda \vec{\nu}\|_{L^{2}(\partial \Omega)} \\
& \leq\|Q \vec{f}\|_{L^{2}(\partial \Omega)}+C\|\vec{f}-\lambda \vec{\nu}\|_{L^{2}(\partial \Omega)} \\
& \leq\|Q \vec{f}\|_{L^{2}(\partial \Omega)}+C\|S(\vec{f}-\lambda \vec{\nu})\|_{L_{1}^{2}(\partial \Omega)} \\
& =\|Q \vec{f}\|_{L^{2}(\partial \Omega)}+C\|S \vec{f}\|_{L_{1}^{2}(\partial \Omega)},
\end{aligned}
$$

where the next-to-last inequality relies on (6.24), while the last one uses (2.24). This finishes the proof of the lemma.

We are now ready to state and prove the analogue of Proposition 5.1 for bounded domains. To set the stage, much as in (3.2), we define the operator

$$
T_{r}: L^{2}(\partial \Omega) \longrightarrow L_{1}^{2}(D) \oplus L^{2}(N), \quad T_{r} \vec{f}:=\left(\left.(S \vec{f})\right|_{D},\left.\left(\left(-\frac{1}{2} I+K_{r}\right) \vec{f}\right)\right|_{N}\right)
$$

Proposition 6.2. Let $\Omega \subseteq \mathbb{R}^{n}, n \geq 3$, be a Lipschitz creased domain. For each $r \in(-1,1]$ there exists $C>0$ such that

$$
\|\vec{f}\|_{L^{2}(\partial \Omega)} \leq C\left\|T_{r} \vec{f}\right\|_{L_{1}^{2}(D) \oplus L^{2}(N)},
$$

for all $\vec{f} \in L^{2}(\partial \Omega)$.

Proof. Let $\vec{f} \in L^{2}(\partial \Omega)$ be arbitrary and set $\vec{u}:=\mathcal{S} \vec{f}, \pi:=\mathcal{Q} \vec{f}$ in $\Omega$ (so that (3.4) holds). Together, Lemma 6.1, (2.23), (2.29), and (6.20) yield

$$
\begin{aligned}
\|\vec{f}\|_{L^{2}(\partial \Omega)} & \leq C\|S \vec{f}\|_{L_{1}^{2}(\partial \Omega)}+C\|Q \vec{f}\|_{L^{2}(\partial \Omega)} \\
& \leq C\|\vec{u}\|_{L_{1}^{2}(\partial \Omega)}+C\|\pi\|_{L^{2}(\partial \Omega)} \leq C\left\|\partial_{\nu}^{r}(\vec{u}, \pi)\right\|_{L^{2}(N)}+C\|\vec{u}\|_{L_{1}^{2}(D)} \\
& =C\left\|T_{r} \vec{f}\right\|_{L_{1}^{2}(D) \oplus L^{2}(N)},
\end{aligned}
$$

for some finite $C=C(\Omega, D, N, r)>0$.

Now consider the one-parameter family of mixed boundary value problems for the Stokes system in a bounded, Lipschitz domain $\Omega$ :

$$
\left\{\begin{array}{l}
\Delta \vec{u}-\nabla \pi=0 \text { and } \operatorname{div} \vec{u}=0 \text { in } \Omega, \\
\left.\vec{u}\right|_{D}=\vec{f}_{D} \in L_{1}^{2}(D), \\
\left.\left[r(\nabla \vec{u})+(\nabla \vec{u})^{\top}\right] \vec{\nu}-\pi \vec{\nu}\right]\left.\right|_{N}=\vec{f}_{N} \in L^{2}(N), \\
M(\nabla \vec{u}), M(\vec{u}), M(\pi) \in L^{2}(\partial \Omega),
\end{array}\right.
$$

where $r \in \mathbb{R}$ and $D, N$ partition $\partial \Omega$.

Theorem 6.3. Let $\Omega \subset \mathbb{R}^{n}, n \geq 3$, be a bounded creased Lipschitz domain with connected boundary. Then the mixed boundary value problem (6.29) is well-posed for each $r \in(-1,1]$. 
Proof. It has been proved in [9, 21] that for any bounded Lipschitz domain $\Omega$, conditions (3.4) in Lemma 3.2 imply that there exists $\vec{f} \in L^{2}(\partial \Omega)$ such that $\vec{u}=\mathcal{S} \vec{f}$ and $\pi=\mathcal{Q} \vec{f}$ in $\Omega$. With this in hand one can then check, much as in the proof of Theorem 3.3, that

$T_{r}$ in (6.26) is an isomorphism $\Longleftrightarrow$ the mixed problem (6.29) is well-posed.

The fact that the operator $T_{r}$ in (6.26) is an isomorphism can, in turn, be established with the help of Proposition 6.2 and Lemma 5.3 by proceeding as in the proof of Theorem 5.2 and invoking the results in [4].

We conclude with a few remarks.

Remark 1. While here it has been hypothesized that $\partial \Omega$ is connected, for practical applications it is certainly of interest to consider domains with a nontrivial topology. In the case when either $D$ or $N$ is empty, this has recently been done in 21], and it is natural to speculate that some of the ideas developed there could be used to further extend the current results.

Remark 2. Dictated by practical considerations, it is of interest to study boundary value problems for the Stokes system equipped with other types of mixed conditions, such as

$$
\text { prescribing } \vec{u}_{t a n} \text { and }\left\{\left[(\nabla \vec{u})+(\nabla \vec{u})^{\top}\right] \vec{\nu}\right\}_{\text {tan }} \text { on } N \text {, }
$$

in place of the third condition in (1.1).

Remark 3. As already pointed out in the Introduction, it is of interest to determine the optimal range of $p$ 's for which the $L^{p}$-version of (1.1) is well-posed. Prior work in the case of the Laplace operator ([3]) and the Stokes system when either $D=\emptyset$, or $N=\emptyset$ ([21]) suggests that, at least when $n=3$, the current results should have suitable Hardy-space versions whenever $1-\varepsilon<p<2+\varepsilon$.

\section{ACKNOWLEDGMENTS}

Part of this work was carried out while some of the authors were attending the joint AMS-MMS meeting in Zacatecas, Mexico, in the Spring of 2007. We wish to thank the organizers of the Harmonic Analysis Section (which was dedicated to the memory of Misha Cotlar), Salvador Pérez-Esteva and Josefina Alvarez, for putting together an interesting event with a stimulating atmosphere.

\section{REFERENCES}

[1] G. Alessandrini, A. Morassi, E. Rosset, The linear constraints in Poincaré and Korn type inequalities, Forum Math., 20 (2008), no. 3, 557-569. MR2418206 (2009b:26019)

[2] M.E. Bogovskiı̌, Solutions of some problems of vector analysis, associated with the operators div and grad, Trudy Sem. S.L. Soboleva, No. 1, 1980, Akad. Nauk SSSR Sibirsk. Otdel., Inst. Mat., Novosibirsk, 1980, pp. 5-40. MR631691 (82m:26014)

[3] R.M. Brown, The mixed problem for Laplace's equation in a class of Lipschitz domains, Comm. Partial Diff. Eqns., 19 (1994), 1217-1233. MR1284808 (95i:35059)

[4] R.M. Brown and I. Mitrea, The mixed problem for the Lamé system in a class of Lipschitz domains, J. Differential Equations, 246 (2009), no. 7, 2577-2589. MR2503013

[5] R. Coifman, A. McIntosh and Y. Meyer, L'intégrale de Cauchy définit un opérateur borné sur $L^{2}$ pour les courbes lipschitziennes, Ann. of Math. (2), 116 (1982), no. 2, 361-387. MR672839 (84m:42027)

[6] P.G. Ciarlet, Three-Dimensional Elasticity, Vol. 1, Elsevier Science Publishers, 1998. 
[7] B.E.J. Dahlberg, C.E. Kenig, and G. Verchota, Boundary value problems for the systems of elastostatics in Lipschitz domains, Duke Math. J., 57 (1988), 795-818. MR.975122 (90d:35259)

[8] E. B. Fabes, M. Jodeit and N. Rivière, Potential techniques for boundary value problems on $C^{1}$ domains, Acta Math., 141 (1978), 165-186. MR501367 (80b:31006)

[9] E.B. Fabes, C.E. Kenig and G.C. Verchota, The Dirichlet problem for the Stokes system on Lipschitz domains, Duke Math. J., 57 (1988), no. 3, 769-793. MR975121 (90d:35258)

[10] P. Grisvard, Elliptic Problems in Nonsmooth Domains, Monographs and Studies in Mathematics, Vol. 24, Pitman, Boston, MA, 1985. MR775683 (86m:35044)

[11] H.Ito, Optimal Korn's inequalities for divergence-free vector fields with applications to incompressible linear elastodynamics, Japan J. Indust. Appl. Math., 16 (1999), 101-121. MR.1676350 (2000i:74010)

[12] C.E. Kenig, Harmonic Analysis Techniques for Second Order Elliptic Boundary Value Problems, CBMS Regional Conference Series in Mathematics, Vol.83, American Mathematical Society, Providence, RI, 1994. MR1282720 (96a:35040)

[13] M. Kohr, A mixed boundary value problem for the unsteady Stokes system in a bounded domain in $\mathbb{R}^{n}$, Engineering Analysis with Boundary Elements, 29 (2005), 936-943.

[14] L. Lanzani, L. Capogna, and R.M. Brown, The mixed problem in $L^{p}$ for some twodimensional Lipschitz domains, Math. Ann., 342 (2008), no. 1, 91-124. MR2415316 (2009c:35051)

[15] V. Maz'ya and J. Rossmann, Mixed boundary value problems for the Navier-Stokes system in polyhedral domains, preprint, 2006.

[16] I. Mitrea and M. Mitrea, The Poisson problem with mixed boundary conditions in Sobolev and Besov spaces in nonsmooth domains, Trans. Amer. Math. Soc., 359 (2007), 4143-4182. MR.2309180 (2008e:35031)

[17] D. Mitrea, M. Mitrea and S. Monniaux, The Poisson problem for the exterior derivative operator with Dirichlet boundary condition on nonsmooth domains, Commun. Pure Appl. Anal., 7 (2008), no. 6, 1295-1333. MR2425010

[18] M. Mitrea, Mixed boundary-value problems for Maxwell's equations, to appear in the Transactions of the American Mathematical Society, 2007.

[19] J. Nečas, Les Méthodes Directes en Théorie des Équations Elliptiques, Masson et Cie, Éditeurs, Paris, Academia, Éditeurs, Prague, 1967. MR0227584 (37:3168)

[20] J.D. Sykes and R. M. Brown, The mixed boundary problem in $L^{p}$ and Hardy spaces for Laplace's equation on a Lipschitz domain, pp.1-18 in "Harmonic Analysis and Boundary Value Problems" Contemp. Math., Vol. 277, Amer. Math. Soc., Providence, RI, 2001. MR.1840423 (2002g:35058)

[21] M. Wright and M. Mitrea, The transmission problem for the Stokes system in Lipschitz domains, preprint, 2007.

Department of Mathematics, University of Kentucky, Lexington, Kentucky 40506

E-mail address: russell.brown@uky.edu

Department of Mathematical Sciences, Worcester Polytechnic Institute, WorcesTER, Massachusetts 01609

E-mail address: imitrea@wpi.edu 65211

Department of Mathematics, University of Missouri at Columbia, Columbia, Missouri

E-mail address: marius@math.missouri.edu

Department of Mathematics, University of Missouri at Columbia, Columbia, Missouri 65211

E-mail address: wrightm@math.missouri.edu 